\title{
Competência glótica na doença pulmonar obstrutiva crônica
}

\section{Glottal competence in chronic obstructive pulmonary disease}

\author{
Rachel de Aguiar Cassiani', Lílian Aguiar-Ricz², Carla Manfredi dos Santos', José Antonio Baddini Martinez \\ Roberto Oliveira Dantas ${ }^{3}$
}

\section{RESUMO}

Objetivo: Correlacionar a medida do tempo máximo de fonação (TMF) com o volume de ar pulmonar expirado no primeiro segundo do sopro $\left(V_{E F}\right)$ e comparar o TMF, emissão do "s", "z" e relação s/z em indivíduos saudáveis e indivíduos com doença pulmonar obstrutiva crônica (DPOC). Métodos: Foram avaliados 14 voluntários saudáveis, com média de idade de 65 anos, e 16 indivíduos com DPOC, com média de idade de 68 anos. O diagnóstico da doença foi clínico e também obtido por exames da função pulmonar. Os participantes foram submetidos à espirometria para apreciação dos parâmetros $\mathrm{VEF}_{1}$, capacidade vital forçada (CVF) e o índice de Tiffeneau $\left(\mathrm{VEF}_{1} / \mathrm{CVF}\right)$. Registrou-se a emissão prolongada das vogais "a”, "i”, "u” para análise do tempo máximo de fonação na condição normal e forçada e aferiu-se a relação s/z após a sustentação dos fonemas /s/ e /z/. Resultados: Nos dois grupos, o TMF com a fonação normal foi menor do que o tempo máximo de fonação, com a fonação forçada. A mediana do TMF do grupo controle foi maior do que a do grupo com DPOC, com a fonação normal e a fonação forçada. Não houve correlação entre o tempo máximo de fonação e os valores de $\mathrm{VEF}_{1}$ nos grupos. Conclusão: Pacientes com DPOC tem diminuição do tempo máximo de fonação e a relação s/z sugere falta de coaptação glótica. Não houve correlação do TMF e a medida do $\mathrm{VEF}_{1}$.

Descritores: Laringe; Doença Pulmonar Obstrutiva Crônica; Fonação; Voz; Disfonia

\begin{abstract}
Purpose: This study correlates the extent of maximum phonation time (MPT) with the volume of air exhaled in the first second pulmonary $\left(\mathrm{FEV}_{1}\right)$ and compares the MPT emission of /s/, /z/ and s/z ratio in healthy subjects and those with chronic obstructive pulmonary disease (COPD). Methods: We studied 14 normal volunteers (control group), mean age 65 years, and 16 patients with COPD, mean age 68 years. The diagnosis of COPD was done by clinical and pulmonary function evaluation. Participants underwent spirometry for analysis of the parameters $\mathrm{FEV}_{1}$, forced vital capacity (FVC) and Tiffeneau index $\left(\mathrm{FEV}_{1} / \mathrm{FVC}\right)$. The maximum phonation time, along with phonemes $/ \mathrm{s} /$ and $/ \mathrm{z} / \mathrm{s} / \mathrm{z}$ ratio correlation, and maximum phonation time in both normal and forced phonation were collected and recorded. Results: In both groups, the maximum phonation time with normal phonation was lower than the maximum phonation time with forced phonation. The median of the maximum phonation time in the control group was higher than in the COPD group. There was no correlation between the maximum phonation time and $\mathrm{FEV}_{1}$, in normal volunteers and patients with COPD. Conclusion: Patients with COPD have decreased maximum phonation time, while the s/z ratio does not suggest changes in the level of the vocal folds, since the values found were within the normal range. There was no correlation of the TMF and the measure of $\mathrm{FEV}_{1}$.
\end{abstract}

Keywords: Larynx; Pulmonary Disease, Chronic Obstructive; Phonation; Voice; Dysphonia

Trabalho realizado nos Departamentos de Clínica Médica e de Oftalmologia, Otorrinolaringologia e Cirurgia de Cabeça e Pescoço, Faculdade de Medicina de Ribeirão Preto, Universidade de São Paulo - USP - Ribeirão Preto (SP), Brasil.

(1) Centro Integrado de Reabilitação do Hospital Estadual de Ribeirão Preto - Ribeirão Preto (SP), Brasil.

(2) Departamento de Oftalmologia, Otorrinolaringologia e Cirurgia de Cabeça e Pescoço, Faculdade de Medicina de Ribeirão Preto, Universidade de São Paulo - USP - Ribeirão Preto (SP), Brasil.

(3) Departamento de Clínica Médica, Faculdade de Medicina de Ribeirão Preto, Universidade de São Paulo - USP - Ribeirão Preto (SP), Brasil.

Conflito de interesses: Não

Contribuição dos autores: $R A C$ participou do planejamento, obtenção dos resultados, análise e interpretação dos dados, redação do manuscrito e na decisão em submetê-lo para publicação; $L A R$ participou do planejamento, análise e interpretação dos dados, redação do manuscrito e na decisão em submetê-lo para publicação; $C M S$ participou do planejamento, análise e interpretação dos dados, redação do manuscrito e na decisão em submetê-lo para publicação; JABM Participou do planejamento, análise e interpretação dos dados, redação do manuscrito e na decisão em submetê-lo para publicação; ROD, pesquisador principal, participou do planejamento, obtenção dos resultados, análise e interpretação dos dados, redação do manuscrito e na decisão em submetê-lo para publicação.

Endereço para correspondência: Roberto Oliveira Dantas. Universidade de São Paulo, Departamento de Clínica Médica, Faculdade de Medicina de Ribeirão Preto. Av. Bandeirantes, 3900, Monte Alegre, Ribeirão Preto (SP), Brasil, CEP: 14048-900. E-mail: rodantas@ fmrp.usp.br

Recebido em: 5/10/2012; Aceito em: 12/8/2013 


\section{INTRODUÇÃO}

A doença pulmonar obstrutiva crônica (DPOC) é uma enfermidade comum, geralmente progressiva e debilitante, caracterizada por sinais e sintomas respiratórios associados à limitação da capacidade ventilatória, sendo geralmente causada por exposição inalatória crônica a material particulado, principalmente decorrente de tabagismo ${ }^{(1,2)}$. A diminuição do volume pulmonar promove diminuição da pressão subglótica e compromete a coaptação glótica da laringe, durante a fonação. A condição respiratória modifica a fonação, embora essa relação ainda não tenha sido demonstrada experimentalmente ${ }^{(3)}$.

O diagnóstico da DPOC é confirmado pela espirometria, com obtenção da curva expiratória volume-tempo, de preferência em fase estável da doença. A espirometria permite a avaliação de uma multiplicidade de parâmetros, porém os mais importantes, do ponto de vista de aplicação clínica, são o $\mathrm{VEF}_{1}$ (volume expiratório forçado no primeiro segundo), a CVF (capacidade vital forçada) e a relação $\mathrm{VEF}_{1} / \mathrm{CVF}$, pois mostram menor variabilidade inter e intraindividual. A existência de limitação do fluxo aéreo é definida pela presença da relação $\mathrm{VEF}_{1} / \mathrm{CVF}$, abaixo de $0,70^{(1,4)}$.

Segundo a Sociedade Brasileira de Pneumologia e Tisiologia (SBPT), a prevalência de DPOC no Brasil é de $15,8 \%$ em adultos acima de $40 \mathrm{anos}^{(1)}$. O sintoma mais frequente é a tosse, que pode ser diária ou intermitente e pode preceder a dispneia, ou aparecer simultaneamente a ela. A capacidade ventilatória reduzida, a fraqueza dos músculos intercostais e abdominais, juntamente com a presença da dispneia são os fatores responsáveis pela limitação das atividades cotidianas dos indivíduos com $\mathrm{DPOC}^{(5)}$. O aumento da carga de trabalho dos músculos respiratórios, do consumo de oxigênio e o maior gasto energético ocorrem na tentativa de superar a obstrução do fluxo livre do ar, principalmente durante a expiração( ${ }^{(6)}$, o que pode favorecer a incoordenação da respiração, da fonação e do desempenho da comunicação oral do indivíduo.

A comunicação oral é o meio pelo qual o indivíduo relata experiências, ideias, conhecimentos e sentimentos e o indivíduo portador de DPOC poderá ter dificuldade em se comunicar, pois tarefas fonatórias impõe exigências respiratórias consideráveis para o desempenho da fala, tanto em termos de fluxo expiratório como em termos de excursões de expiração do volume pulmonar ${ }^{(7)}$.

Apesar do entendimento que a fonação pode ser alterada por doença pulmonar, não há evidências científicas de medidas objetivas e tampouco de realização de provas fonatórias na avaliação clínica de rotina de pacientes com $\operatorname{DPOC}^{(8)}$.

O parâmetro de avaliação clínica para análise dos componentes fonatórios e respiratórios do mecanismo de produção da voz e da fala é o tempo máximo de fonação (TMF) ${ }^{(9,10)}$. Outro teste que permite avaliar a habilidade do indivíduo em controlar as forças aerodinâmicas da corrente pulmonar e as forças mioelásticas da laringe é a emissão sustentada dos fonemas fricativo surdo "s" e sonoro " $\mathrm{z}$ "(11).

O conhecimento da condição fonatória nos indivíduos portadores de DPOC poderá favorecer o melhor desempenho da comunicação oral. Embora a fonação e, por sua vez, a produção da fala, possam ser alteradas por doença pulmonar, não foram observadas referências na literatura especializa$\mathrm{da}^{(7,8)}$. Diante disso, o objetivo deste estudo foi correlacionar a medida do tempo máximo de fonação (TMF) com o volume de ar pulmonar expirado no primeiro segundo do sopro $\left(V F_{1}\right)$ e comparar o TMF, emissão do "s", "z" e relação s/z em indivíduos saudáveis e indivíduos com doença pulmonar obstrutiva crônica (DPOC).

\section{MÉTODOS}

Este estudo foi aprovado pelo Comitê de Ética em Pesquisa do Hospital das Clínicas da Faculdade de Medicina de Ribeirão Preto, da Universidade de São Paulo (USP), processo número 9473/2005. Todos os indivíduos assinaram o Termo de Consentimento Livre e Esclarecido, para participação na pesquisa. $\mathrm{O}$ tipo de estudo foi prospectivo transversal.

Foram estudados 16 indivíduos com DPOC, com idades entre 56 e 77 anos, média de 68,4 anos, sendo um do gênero feminino $(6,2 \%)$ e 15 do gênero masculino $(93,8 \%)$. Foram estabelecidos os seguintes critérios de inclusão no estudo: pacientes com diagnóstico de DPOC em fase estável da doença, assintomáticos de tosse e dispneia e sem indicação para uso de medicação DPOC classificada como moderada a muito grave, de acordo com exame de espirometria; pacientes com história relevante de tabagismo no passado, mas que tinham abandonado o vício há, pelo menos, seis meses.

Foram excluídos indivíduos com antecedentes de outras doenças pulmonares crônicas e portadores de outras moléstias significativas, tais como: cardiopatias graves, neoplasias, diabetes, ou hipertensão arterial grave, além de história pregressa e/ou atual de disfonia.

O grupo controle foi composto por 14 indivíduos com idades entre 57 e 73 anos, média de 65,3 anos, sendo três do gênero feminino (20\%) e 11 do gênero masculino (80\%), todos com exame de espirometria normal, que nunca foram tabagistas ou que fumaram menos de 10 anos/maço, valor definido pelo total de anos de fumo multiplicado pelo total de maços de cigarro ao dia. Não foram aceitos indivíduos com antecedentes de outras doenças pulmonares crônicas e portadores de outras moléstias significativas, tais como: cardiopatias graves, neoplasias, diabetes, ou hipertensão arterial grave, além de história pregressa e/ou atual de disfonia. Aqueles que haviam sido fumantes no passado, tinham abandonado o hábito há, pelo menos, um ano. Tanto os indivíduos do grupo controle como os do grupo com DPOC não relataram queixas vocais. 


\section{Procedimentos}

\section{Espirometria}

A caracterização do diagnóstico e da gravidade da DPOC foi realizada pelo pneumologista, por meio de análise da espirometria completa, e os dois grupos do estudo foram submetidos a essa avaliação. As espirometrias foram realizadas em um espirômetro Pulmonet III ${ }^{\circledR}$ (Sensormedics, Anaheim, CA, USA), conforme a padronização da Sociedade Brasileira de Pneumologia e Tisiologia ${ }^{(4)}$.

Para obtenção das curvas pela espirometria, os indivíduos permaneceram na posição sentada. A prova foi executada com o bocal do espirômetro acoplado e com uma presilha nasal, para que não houvesse fuga de ar. Os indivíduos receberam orientação sobre manobras a serem realizadas e foram estimulados, de forma vigorosa pelo examinador, a inspirar até encher totalmente os pulmões, segurar o ar, colocar os lábios em torno do bocal limpo e, imediatamente após, assoprar o ar o mais rapidamente possível e com o máximo de esforço, prolongando o sopro até sentir os pulmões plenamente vazios.

\section{Análise dos índices espirométricos}

A partir de curvas consideradas como de qualidade técnica aceitável, foram calculados os seguintes parâmetros para o diagnóstico e classificação do grau de severidade da DPOC: volume expiratório forçado no primeiro segundo $\left(\mathrm{VEF}_{1}\right)$, capacidade vital forçada (CVF) e o índice de Tiffeneau $\left(\mathrm{VEF}_{1} / \mathrm{CVF}\right)$. O cálculo dos valores previstos para as variáveis da espirometria foi realizado empregando-se as equações de Pereira et $\mathrm{al}^{(12)}$. Os resultados dos parâmetros da espirometria foram expressos como percentual do previsto, conforme preconizado pelo II Consenso de DPOC da Sociedade Brasileira de Pneumologia e Tisiologia ${ }^{(4)}$.

\section{Padrões de índices espirométricos}

- VEF1: Volume expiratório forçado no primeiro segundo: $o$ volume de ar expirado no primeiro segundo do sopro;

- CVF: Capacidade vital forçada: volume total de ar que pode ser forçadamente expirado em uma respiração;

- Relação VEF1/CVF ou índice de Tiffeneau ( $\left(\mathrm{VEF}_{1} / \mathrm{CVF}\right)$ : fração de ar expirado no primeiro segundo, relativo ao volume total expirado.

\section{Avaliação clínica vocal do controle das forças aerodinâmicas e das forças mioelásticas da laringe}

Para a avaliação, aplicou-se a prova do tempo máximo de fonação das vogais prolongadas e a emissão sustentada das fricativas surda "s" e sonora "z" e a relação s/z, por meio da medição do fluxo de ar durante a fonação, expressas em segundos ${ }^{(13)}$.

Solicitou-se ao participante, após inspiração profunda, que emitisse as vogais /a/, /i/, /u/ e os fonemas /s/ e /z/, de forma prolongada, e que não utilizasse $\mathrm{o}$ ar residual durante a aplicação da prova de fonação. A frequência e a intensidade da voz deveriam ser habituais, indicando naturalidade e caracterizando a fonação normal. A seguir, o mesmo procedimento foi repetido, porém o indivíduo foi orientado a utilizar todo o ar pulmonar de reserva, caracterizando a fonação forçada.

\section{Padrões da avaliação clínica vocal do controle das forças aerodinâmicas e das forças mioelásticas da laringe}

Solicitou-se a emissão prolongada da vogal /a/ por ser oral, central e aberta e que, por sua natureza articulatória, pode evidenciar quaisquer alterações no equilíbrio mioelástico da laringe. A vogal /i/ é a mais aguda do português e a mais fechada anteriormente, enquanto a vogal /u/ é a mais grave e a mais fechada posteriormente ${ }^{(13)}$.

A emissão prolongada do fonema fricativo surdo /s/ permite verificar o suporte aéreo pulmonar e a habilidade de controlá-lo, uma vez que não há vibração de pregas vocais. Já a emissão da consoante sonora /z/, requer vibração de pregas vocais ${ }^{(13)}$.

Os participantes mantiveram-se confortavelmente sentados, sem comprimir a musculatura diafragmática, com os pés apoiados no chão e mensurou-se o TMF de cada uma das vogais e dos fonemas em segundos, utilizando-se um cronômetro digital (Technos ${ }^{\circledR}$, Amazônia, Brasil).

\section{Análise dos resultados da avaliação clínica vocal do controle das forças aerodinâmicas e das forças mioelásticas da laringe}

Utilizou-se, para análise, os valores de normalidade validados para a população brasileira, considerando-se 20 segundos para homens, 14 segundos para mulheres e a relação s/z, entre 0,8 segundos e 1,2 segundos, definidos como valores de normalidade expressos em segundos, para indivíduos adultos ${ }^{(13)}$.

Os resultados do tempo máximo de fonação, emissão do "s" e "z" e relação s/z foram comparados entre os grupos, aplicando-se dois testes não paramétricos: o teste de Wilcoxon (para amostras dependentes) e o teste de Mann-Whitney (para amostras independentes), com utilização do software $R^{\circledR}$. Para correlacionar os parâmetros avaliados, foram considerados o tempo máximo de fonação e o volume expiratório forçado no primeiro segundo $\left(\mathrm{VEF}_{1}\right)$. Para tanto, calculou-se o coeficiente de correlação de Pearson (r), utilizando-se o mesmo software. Em todas as análises estatísticas, adotou-se o nível de significância de $5 \%(\mathrm{p} \leq 0,05)$.

\section{RESULTADOS}

O resultado da espirometria permitiu observar os valores do índice de Tiffeneau $\left(\mathrm{VEF}_{1} / \mathrm{CFV}\right)$ de $42,5 \pm 10,1 \%$ para o grupo DPOC e de $76,9 \pm 5,8 \%$ para o grupo controle. Verificou-se limitação do fluxo aéreo para o grupo DPOC, que foi definido como abaixo de $0,70 \%$, pela relação $\mathrm{VEF}_{1} / \mathrm{CVF}$.

$\mathrm{O}$ valor previsto de normalidade para o $\mathrm{VEF}_{1}$ é de $\geq 80 \%$ e o 
resultado encontrado para o grupo com DPOC foi de 40,2 $\pm 8,1 \%$ e para o grupo controle foi de $98,5 \pm 12,6 \%$. Segundo esses critérios, os pacientes com DPOC apresentaram a relação $\mathrm{VEF}_{1} / \mathrm{CVF}$ abaixo do limite inferior da normalidade e o grupo controle, acima do limite inferior, conforme dados balizados no II Consenso de DPOC da Sociedade Brasileira de Pneumologia e Tisiologia ${ }^{(4)}$.

Foi observada diferença no TMF entre o grupo controle e os pacientes com DPOC, tanto quando avaliados com fonação normal, quanto na avaliação com a fonação forçada. O TMF foi mais longo no grupo controle do que no grupo de indivíduos com DPOC, com a fonação normal (Tabela 1) e com a fonação forçada (Tabela 2).

No grupo controle, a relação s/z durante a fonação normal teve mediana de 0,8 segundos (mínimo de 0,6 s e máximo de 1,7 s) e no grupo com DPOC, a mediana foi de 1,3 segundos (mínimo de 0,7 s e máximo $2,3 \mathrm{~s}$ ), com diferença significativa entre os grupos $(\mathrm{p}=0,04)$.

Quando se avaliou a correlação entre a medida do tempo máximo de fonação e a medida pelo $\mathrm{VEF}_{1}$, não se observou correlação entre as variáveis, nem no grupo controle, nem no grupo de pacientes com DPOC, tanto na fonação normal quanto na forçada (Tabela 3).

\section{DISCUSSÃO}

Os indivíduos com doença obstrutiva de vias aéreas, em função da alta resistência ao fluxo de ar, possuem capacidade

Tabela 1. Comparação da mediana do tempo máximo de fonação (TMF), em segundos (s), entre o grupo de indivíduos com DPOC e o grupo controle, em fonação normal

\begin{tabular}{|c|c|c|c|c|c|}
\hline & \multicolumn{2}{|c|}{ Controle } & \multicolumn{2}{|c|}{ DPOC } & \multirow{2}{*}{ Valor de $p$} \\
\hline & Mediana & Mínimo-máximo & Mediana & Mínimo-máximo & \\
\hline Vogal /a/ & $12 \mathrm{~s}$ & $6-20 \mathrm{~s}$ & $7 \mathrm{~s}$ & $2-12 s$ & $<0,01^{*}$ \\
\hline Vogal /i/ & $13 s$ & $7-29 \mathrm{~s}$ & $7 \mathrm{~s}$ & $3-12 \mathrm{~s}$ & $<0,01^{*}$ \\
\hline Vogal /u/ & $14 \mathrm{~s}$ & $7-23 s$ & $7 s$ & $3-12 s$ & $<0,01^{*}$ \\
\hline Fonema /s/ & $11 \mathrm{~s}$ & $5-18 s$ & $8,5 \mathrm{~s}$ & $4-11 s$ & $0,03^{*}$ \\
\hline Fonema /z/ & $13 s$ & $5-21 s$ & $6,5 \mathrm{~s}$ & $3-14 s$ & $<0,01^{*}$ \\
\hline Relação s/z & $0,8 \mathrm{~s}$ & & $1,3 \mathrm{~s}$ & & \\
\hline
\end{tabular}

*Valores significativos $(p \leq 0,05)$ - Teste de Man-Whitney

Legenda: DPOC = doença pulmonar obstrutiva crônica

Tabela 2. Comparação da mediana do tempo máximo de fonação (TMF) em segundos (s), entre o grupo de indivíduos com DPOC e o grupo controle, em fonação forçada

\begin{tabular}{|c|c|c|c|c|c|}
\hline & \multicolumn{2}{|c|}{ Controle } & \multicolumn{2}{|c|}{ DPOC } & \multirow{2}{*}{ Valor de $p$} \\
\hline & Mediana & Mínimo-máximo & Mediana & Mínimo-máximo & \\
\hline Vogal /a/ & $18 \mathrm{~s}$ & $9-38 s$ & $10,5 \mathrm{~s}$ & $2-17 \mathrm{~s}$ & $<0,01^{*}$ \\
\hline Vogal /i/ & $23 \mathrm{~s}$ & $6-35 s$ & $11 \mathrm{~s}$ & $2-21 s$ & $<0,01^{*}$ \\
\hline Vogal /u/ & $16 \mathrm{~s}$ & $11-39 \mathrm{~s}$ & $11 \mathrm{~s}$ & $4-17 \mathrm{~s}$ & $<0,01^{*}$ \\
\hline Fonema /s/ & $15 \mathrm{~s}$ & $8-27 \mathrm{~s}$ & $12 \mathrm{~s}$ & $5-17 \mathrm{~s}$ & $0,04^{*}$ \\
\hline Fonema /z/ & $18 \mathrm{~s}$ & $8-33 \mathrm{~s}$ & $8,5 \mathrm{~s}$ & $4-18 s$ & $<0,01^{*}$ \\
\hline
\end{tabular}

*Valores significativos $(p \leq 0,05)$ - Teste de Man-Whitney

Legenda: DPOC = doença pulmonar obstrutiva crônica

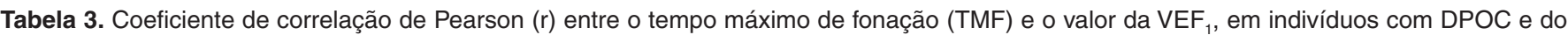
grupo controle, em fonação normal e fonação forçada

\begin{tabular}{|c|c|c|c|c|c|c|c|c|}
\hline & \multicolumn{4}{|c|}{ Controle } & \multicolumn{4}{|c|}{ DPOC } \\
\hline & \multicolumn{2}{|c|}{ Normal } & \multicolumn{2}{|c|}{ Forçada } & \multicolumn{2}{|c|}{ Normal } & \multicolumn{2}{|c|}{ Forçada } \\
\hline & $r$ & $\mathrm{p}$ & $r$ & $p$ & $r$ & $\mathrm{p}$ & r & $\mathrm{p}$ \\
\hline $\mathrm{TMF}$ Vogal /a/ X VEF & $-0,16$ & 0,62 & 0,18 & 0,58 & $-0,06$ & 0,83 & 0,43 & 0,10 \\
\hline TMF Vogal /i/ X VEF & $-0,15$ & 0,64 & 0,09 & 0,79 & 0,38 & 0,15 & 0,34 & 0,20 \\
\hline TMF Vogal /u/ X VEF ${ }_{1}$ & $-0,27$ & 0,39 & $-0,02$ & 0,96 & 0,41 & 0,11 & 0,35 & 0,19 \\
\hline $\mathrm{TMF}$ Fonema $/ \mathrm{s} / \mathrm{XVEF}$ & $-0,55$ & 0,07 & $-0,27$ & 0,39 & $-0,10$ & 0,71 & 0,13 & 0,62 \\
\hline TMF Fonema /z/ X VEF & $-0,32$ & 0,31 & 0,01 & 0,97 & $-0,26$ & 0,33 & 0,03 & 0,93 \\
\hline
\end{tabular}

Coeficiente de correlação de Pearson ( $r)$ - $(p \leq 0,05)$

Legenda: $\mathrm{DPOC}=$ doença pulmonar obstrutiva crônica 
ventilatória limitada, intensificando o trabalho da respiração, com frequências respiratórias altas, em repouso ${ }^{(14)}$. Na literatura pesquisada, não foram encontrados estudos que debatessem a dinâmica fonatória e a competência glótica em DPOC. Por meio dos resultados encontrados neste estudo, infere-se que os pacientes necessitaram de inúmeras recargas aéreas para a manutenção e o término da frase durante a conversação, já que o tempo máximo de fonação apresentado pelos pacientes foi significativamente mais baixo do que o do grupo controle, revelando-se inferiores aos valores de normalidade para população adulta $^{(13)}$. Tarefas fonatórias impõem exigências respiratórias consideráveis, tanto em termos de fluxo expiratório quanto em termos de excursões de expiração do volume pulmonar e isso deve ser considerado para todos os indivíduos que dependem da fala para sua subsistência, inclusive os com DPOC ${ }^{(2)}$.

Infere-se que a diminuição do tempo máximo de fonação, nos pacientes deste estudo, denotou o comprometimento da coordenação respiratória, fônica e articulatória, podendo influenciar diretamente o desempenho da comunicação oral e a própria inteligibilidade do discurso dos indivíduos com DPOC. Esse raciocínio encontra-se apoiado no conhecimento de que o volume pulmonar é importante para a sustentação das tarefas fonatórias. Os indivíduos saudáveis podem manter o fluxo de ar expiratório, enquanto pacientes com obstrução grave têm dificuldades, em função da limitação obstrutiva do fluxo aéreo pulmonar ${ }^{(2,3)}$.

Para os pacientes com DPOC, que utilizam a comunicação oral para a maioria das atividades sociais, a avaliação da atividade de fala pode fornecer critérios de medição relevantes e promover melhor desempenho da comunicação oral ${ }^{(8)}$. Para superar essa desvantagem, foi proposto um questionário sobre fala durante as atividades físicas e percebeu-se alta correlação das respostas do questionário no desempenho da fala e atividades de vida diária de indivíduos com DPOC ${ }^{(15)}$, concluindo-se que há melhora das atividades da fala após um programa de reabilitação respiratória em pacientes com DPOC. Recomendase acrescentar tarefas fonatórias validadas e objetivas na avaliação dos sujeitos com DPOC, pois a dispneia interfere na comunicação desses indivíduos ${ }^{(8)}$.

Tendo em vista que não houve correlação do valor de $\mathrm{VEF}_{1}$ com o tempo máximo de fonação, é relevante considerar que os indivíduos com DPOC apresentam o pulmão débil e, provavelmente em função da faixa etária, infere-se que exista presbilaringe com flacidez de pregas vocais e fechamento glótico incompleto.

Para a medida fonatória temporal é necessária a produção prolongada da vogal, sem interferência da configuração glótica e, para o teste da limitação obstrutiva do fluxo aéreo pulmonar, poderia utilizar-se a repetição do "pi" ou "pa", o que exige um desempenho laríngeo de contrarresistência glótica à passagem aérea pulmonar para a produção do fonema e não avalia o fluxo aéreo contínuo. Essas observações são evidenciadas, também, em estudos aeromecânicos acústicos, que mostram diferentes níveis do volume pulmonar em diferentes atividades laríngeas, como leitura de palavras ${ }^{(16)}$ e movimentação vertical da larin$\mathrm{ge}^{(3)}$. Autores reforçam a necessidade de evidências cientificas sobre as medidas objetivas de fonação a serem utilizadas na clínica em pacientes com DPOC ${ }^{(8)}$.

A aplicação da prova clínica da relação s/z tem por excelência verificar a competência das forças mioelásticas da laringe e a aerodinâmica pulmonar. Valores abaixo do limite inferior podem indicar hiperconstrição glótica e valores acima do limite superior são indicativos de falta de coaptação glótica ${ }^{(11)}$.

A diminuição do fluxo aéreo pulmonar na prova do TMF também foi encontrada no grupo controle, provavelmente em virtude da senilidade. Talvez fosse necessário considerar a idade para a interpretação dos resultados do tempo máximo de fonação. Entretanto, atualmente, os dados normativos do TMF estão disponíveis para a população infantil e adulta ${ }^{(13)}$. Portanto, acreditamos que sejam necessárias novas pesquisas para definir os valores de normalidade do TMF para a população de idosos.

O grupo com DPOC evidenciou rebaixamento ainda mais significativo na prova do TMF, gerando fluxo aéreo insuficiente para a promoção do fenômeno de Bernoulli, durante o ciclo glótico. Esse acontecimento faz com que a pressão aérea subglótica sugue as pregas vocais em direção à luz laríngea, favorecendo a coaptação glótica para o ajuste da intensidade e da frequência do som necessário para a produção da fala, conforme preconizado pela teoria mioelástica-aerodinâmica da laringe ${ }^{(17)}$.

O volume pulmonar afeta a fonação e sua diminuição aumenta a capacidade de adução das pregas vocais, enquanto que a pressão subglótica também aumenta e, com isso, a competência glótica tende a melhorar ${ }^{(3)}$. Outra consideração relevante, é a de que o volume pulmonar é um fator que está diretamente relacionado com o posicionamento laríngeo, durante a fonação. Um alto volume pulmonar permite uma posição laríngea baixa, alterando também a frequência da voz ${ }^{(18)}$.

O tempo de latência de voz é maior durante a produção de um fonema surdo com alto volume pulmonar ${ }^{(19)}$. O resultado da presente pesquisa não permite revelar diferença do volume pulmonar no grupo com DPOC, comparado ao grupo controle, durante a produção do /s/. A relação s/z revelou valores próximos do limite da normalidade ${ }^{(10)}$. Quando os dois grupos foram comparados entre si, foi observado maior valor para essa relação no grupo com DPOC, o que sugere fraqueza muscular em nível glótico nesses pacientes. Os valores encontrados nos levaram a inferir que houve ausência de competência glótica ${ }^{(10)}$ e que deve-se rever a queixa e incluir a avaliação da função laríngea dos indivíduos com DPOC.

As perturbações do fluxo expiratório podem ser consequências do uso inadequado da musculatura respiratória, o que ocasiona conflito entre a pressão subglótica e a resistência vocal $^{(10)}$. Acredita-se, portanto, que a obstrução do fluxo livre do ar, principalmente durante a expiração, pode influenciar no TMF e na relação s/z dos pacientes com DPOC, pois a laringe 
une-se mecanicamente ao aparelho respiratório, permitindo que ela sofra influência direta do mesmo.

Diante disso, deve-se considerar a necessidade de se obter medidas objetivas fonatórias, por meio de evidências científicas, para a realização de provas fonatórias na avaliação clínica de rotina de pacientes com DPOC, assim como estabelecer valores de normalidade dos parâmetros clínicos vocais para a população senil, para avaliação e interpretação dos resultados e aplicação de ações fonaudiológicas.

\section{CONCLUSÃO}

Pacientes com DPOC tem diminuição do tempo máximo de fonação, o que pode ser atribuído à limitação obstrutiva do fluxo aéreo pulmonar. A relação s/z sugere alteração na coaptação glótica, durante a fonação, no grupo de indivíduos com DPOC. Não foi observada, entre os grupos estudados, a correlação da medida do tempo máximo de fonação das vogais /a/, /i/, /u/ e os fonemas /s/, /z/ e a medida pelo $\mathrm{VEF}_{1}$.

\section{REFERÊNCIAS}

1. Ministério da Saúde. Departamento de Gestão e Incorporação de Tecnologias em Saúde da Secretaria de Ciência, Tecnologia e Insumos Estratégicos - DGITS/SCTIE. Comissão Nacional de Incorporação de Tecnologias no SUS (CONITEC) - Relatório ${ }^{\circ} 30$.

2. Rabe KF, Hurd S, Anzueto A, Barnes PJ, Buist AS, Calverley P, et al. Global strategy for the diagnosis, management, and prevention of chronic obstructive pulmonary disease: gold executive summary. Am J Respir Crit Care Med. 2007;176(6):532-55.

3. Iwarson J, Sundberg J. Effects of lung volume on vertical larynx position during phonation. J Voice. 1998;12(2):159-65.

4. Jardim JR, Oliveira J, Nascimento O. II Consenso Brasileiro de Doença Pulmonar Obstrutiva Crônica (DPOC). J Bras Pneumol. 2004;30:1-42.

5. Killian KJ, Leblanc P, Martin DH, Summers E, Jones NL, Campbell EJ. Exercise capacity and ventilatory, circulatory, and symptom limitation in patients with chronic airflow limitation. Am Rev Respir Dis. 1992;146(4):935-40.
6. Donahoe M, Rogers RM, Wilson DO, Pennock BE. Oxygen consumption of the respiratory muscles in normal and in malnourished patients with chronic obstructive pulmonary. Am Rev Respir Dis. 1989;140(2):385-91.

7. Binazzi B, Lanini B, Bianchi R, Romagnoli I, Nerini M, Gigliotti $\mathrm{F}$, et al. Breathing pattern and kinematics in normal subjects during speech, singing and loud whispering. Acta Physiol (Oxf). 2006; 186:233-46.

8. Binazzi B, Lanini B, Romagnoli I, Garuglieri S, Stendardi L, Bianchi $\mathrm{R}$, et al. Dyspnea during speech in chronic obstructive pulmonary disease patients: effects of pulmonary rehabilitation. Respiration. 2011;81(5): 379-85.

9. Solomon NP, Garlitz SJ, Milbrath RL. Respiratory and laryngeal contribuitions to maximum phonation duration. J Voice. 2000;14(3):331-40.

10. Cielo CA, Conterno G, Carvalho CDM, Finger LS. Disfonias: relação s/z e tipos de voz. Rev CEFAC. 2008;10(4):536-47.

11. Eckel FC, Boone DR. The S/Z ratio as an indicator of laryngeal pathology. J Speech Hear Disord. 1981;46(2):147-9.

12. Pereira CAC, Barreto SP, Simões JG, Pereira FWL, Gerstler JG, Nakatani J. Valores de referência para espirometria em uma amostra da população brasileira adulta. J Pneumol. 1982;18(1):10-22.

13. Behlau M, Madázio G, Feijó D, Pontes P. Avaliação de voz. In: Behlau M (org). Voz: o livro do especialista. Vol 1. Rio de Janeiro: Revinter; 2005. p.85-120.

14. O’Donnell DE, Revill SM, Webb KA. Dynamic hyperinflation and exercise intolerance in chronic obstructive pulmonary disease. Am J Respir Crit Care Med. 2001;164(5):770-7.

15. Lee L, Friesen M, Lambert IR, Loudon RG. Evaluation of dyspnea during physical and speech activities in patients with pulmonary diseases. Chest. 1998;113:625-32.

16. Milstein CF, Watson PJ. The effects of lung volume initiation on speech: a perceptual study. J Voice. 2004;18(1):38-45.

17. Van Der Berg J. Myoelastic-aerodinamic theory of voice production. J Speech Hear Res. 1958;1(3):227-44.

18. Iwarsson J, Thomasson M, Sundberg J. Effects of lung volume on the glottal voice source. J Voice. 1998;12(4):424-33.

19. Hoit J, Solomon NP, Hixon T. Effect of lung volume on voice onset time (VOT). J Speech Hear Res. 1993;36(3):516-21. 